Relations industrielles

Industrial Relations

\title{
Milkovich, G.T., and A.K. Wigdor, Pay for Performance : Evaluating Performance Appraisal and Merit Pay
}

\section{Roland Thériault}

Volume 48, numéro 1, 1993

URI : https://id.erudit.org/iderudit/050840ar

DOI : https://doi.org/10.7202/050840ar

Aller au sommaire du numéro

Éditeur(s)

Département des relations industrielles de l'Université Laval

ISSN

0034-379X (imprimé)

1703-8138 (numérique)

Découvrir la revue

Citer ce compte rendu

Thériault, R. (1993). Compte rendu de [Milkovich, G.T., and A.K. Wigdor, Pay for Performance : Evaluating Performance Appraisal and Merit Pay]. Relations industrielles / Industrial Relations, 48(1), 190-192.

https://doi.org/10.7202/050840ar

Tous droits réservés @ Département des relations industrielles de l'Université Laval, 1993
Ce document est protégé par la loi sur le droit d'auteur. L’utilisation des services d'Érudit (y compris la reproduction) est assujettie à sa politique d'utilisation que vous pouvez consulter en ligne.

https://apropos.erudit.org/fr/usagers/politique-dutilisation/ 
Pay for Performance: Evaluating Performance Appraisal and Merit Pay, by G.T. Milkovich and A.K. Wigdor, Washington, D.C., National Academy Press, 1991, 210 p., ISBN 0-309-04427-8

À la demande de l'Office of Personnel Management du gouvernement américain, le National Research Council des États-Unis a formé un comité de travail chargé d'étudier les questions d'évaluation du rendement et de la rémunération au mérite pour les fonctionnaires fédéraux américains.

Pay for Performance: Evaluating Performance Appraisal and Merit Pay est le fruit de leur travail. Ce rapport devait contribuer à alimenter les débats lors des audiences du Congrès en vue de l'approbation de la mise à jour du système d'évaluation du rendement et de rémunération au mérite des cadres intermédiaires du gouvernement fédéral, prévue au printemps 1991.

Le comité avait pour mandat de faire une revue critique de la documentation sur le sujet et de procéder à un examen des pratiques existantes dans le secteur privé. De fait, le comité a élargi la portée de son mandat pour inclure, en plus des programmes de rémunération au mérite, tous les programmes de rémunération au rendement individuel et collectif de même que les conditions de succès de ces prorammes.

Outre l'introduction et la conclusion, le contenu de ce livre aurait pu être divisé en trois thèmes: l'histoire de la réforme de la fonction publique fédérale, l'état des recherches et des pratiques en matière d'évaluation du rendement et l'état des recherches et des pratiques en matière de rémunération au mérite. Les auteurs ont préféré le diviser en sept chapitres distincts: 1'histoire de la réforme de la fonction publique fédérale, l'état des connaissances en matière d'évaluation du rendement et de rémunération au rendement, l'évaluation du rendement (définition, mesure et utilité), la rémunération au mérite (concept et résultats de recherches), les pratiques pertinentes dans le secteur privé et l'importance du contexte dans lequel ces pratiques sont mises en place. De plus, à l'instar de tout rapport, un sommaire des résultats des travaux du comité figure au début du livre.

L'histoire de la réforme de la fonction publique fédérale pourra avoir peu d'importance pour certains lecteurs qui ne s'intéressent guère au fonctionnement de l'appareil fédéral en général et américain en particulier. Néanmoins, ce chapitre fait ressortir les principales étapes dans l'histoire de l'évaluation du rendement et de la rémunération au mérite au sein de la fonction publique américaine. À la fin du chapitre, il est question d'un certain nombre d'aspects peu favorables à l'évaluation au rendement et à la rémunération au mérite pour ce type d'employés.

Dès le chapitre 3 , on fait état de la pauvreté relative des recherches en matière d'évaluation du rendement et de rémunération au rendement. Beaucoup a été écrit; peu d'énoncés sont supportés par un ensemble de recherches pertinentes. Cela, cependant, n'empêche pas les auteurs de faire ressortir l'importance de la démarche en matière de mise en œuvre de programmes de gestion des ressources humaines. La façon dont les organisations font les choses est souvent tout aussi importante que ce qu'elles font, sinon plus.

Le chapitre 4 présente les résultats de recherche qui se dégagent des deux principaux courants en matière d'évaluation du rendement, soit le souci de la précision de 
la mesure (courant psychométrique) et celui de l'importance des objectifs de l'évaluation et de son aspect gestion (courant gestion des ressources humaines).

Compte tenu de l'absence de recherche en matière de rémunération au mérite, au chapitre 5, les auteurs élargissent leur champ d'intérêt en faisant un recensement des conclusions qui se dégagent des recherches sur la rémunération au rendement (tant les formules individuelles que collectives). Bien que les effets positifs sur le rendement individuel d'un tel type de régime soient reconnus, on insiste à nouveau sur l'importance du contexte.

Le chapitre 6 présente une synthèse de certaines études effectuées par des sociétés de consultation et des organismes privés au sujet des pratiques d'évaluation du rendement et de rémunération au mérite dans le secteur privé. De plus, on y retrouve les conclusions que les auteurs dégagent de leurs entrevues avec les chefs de ressources humaines de cinq grandes entreprises américaines ainsi que d'une enquête spéciale effectuée par le Conference Board.

Enfin, avant de conclure, le rapport met l'accent sur l'importance de certaines variables contextuelles sur l'efficacité d'un programme d'évaluation du rendement et de rémunération au rendement. On y fait ressortir notamment l'importance de l'harmonie entre, d'une part, l'évaluation et la rémunération et, d'autre part, la nature du travail effectué, la culture et la structure organisationnelles ainsi qu'entre des variables comme le climat économique, la présence ou l'absence d'un syndicat, les pressions attribuables aux lois et l'efficacité des programmes d'évaluation et de rémunération au rendement.

Dans le dernier chapitre, après avoir dégagé les conclusions de son travail, le comité formule ses recommandations. Celles-ci se résument en cinq points dont la portée est générale :

- Il n'y a aucun avantage particulier pour le gouvernement américain à poursuivre ses travaux en vue d'améliorer le caractère psychométrique de son programme d'évaluation du rendement.

- Les programmes efficaces d'évaluation du rendement dans le secteur privé sont ceux qui encouragent les évaluateurs à faire en sorte que l'évaluation produise les résultats désirés. Ainsi, les évaluateurs jouissent d'une certaine flexibilité qui leur permet de reconnaître les personnes qui ont un bon rendement et de se départir des personnes dont le rendement laisse à désirer. Il en est de même en matière de rémunération au mérite. Les gestionnaires peuvent établir des distinctions claires entre les personnes qui ont le meilleur et le moins bon rendement.

- Un programme efficace de rémunération au mérite s'appuie sur des objectifs de travail individuels qui sont réalistes et bien compris. Il existe un lien clair entre l'atteinte de ces objectifs et la rémunération de la personne; le potentiel de rémunération au mérite est significatif.

- La multiplication des procédures et des règlements en vue d'assurer le caractère équitable de la rémunération au mérite a pour effet de décourager les gestionnaires d'utiliser l'évaluation du rendement de facon efficace. 
- Le fait de vouloir modifier un programme d'évaluation du rendement de façon isolée en vue d'en augmenter son acceptation par les gestionnaires et son efficacité peut, à long terme, être aussi coûteux et moins rentable que de procéder à certains changements de nature contextuelle.

Ce rapport ayant été fait en vue de réviser certains aspects de la gestion des ressources humaines de la fonction publique américaine, il est alors fort pertinent pour tout le personnel de la fonction publique intéressé par ces sujets. Il l'est d'ailleurs tout autant pour les personnes du secteur privé.

Ce livre a été rédigé comme un rapport adressé à des preneurs de décisions. Bien qu'on y fasse le point sur l'état des recherches en matière d'évaluation du rendement et de rémunération au mérite, un praticien peut tout aussi bien apprécier son contenu.

Ce document se lit facilement et les messages sont clairs. Toutefois, on a l'impression que les chapitres ont été écrits par des personnes différentes. En effet, il existe certains chevauchements dans les chapitres et, parfois, certaines différences entre les messages. Par exemple, alors qu'au chapitre 6 (page 115), on présente la formule des montants forfaitaires (lump sum) utilisée comme solution de rechange à celle des augmentations de salaire en disant que certaines organisations n'intègrent pas ces montants aux salaires, on dit au chapitre suivant (page 131) que de fait ces montants ne sont pas intégrés au salaire.

A l'occasion, le lecteur averti aurait aimé que l'on nuance un peu les propos ou que l'on soit plus explicite.

Par exemple, on affirme, à la page 78, que la rémunération au mérite est appliquée à l'ensemble du personnel dans les entreprises, des employés de production et de support administratif aux cadres supérieurs. Une telle affirmation mériterait certes d'être nuancée.

Par ailleurs, avant de présenter les résultats de recherche au sujet des régimes de rémunération au rendement, les auteurs les groupent selon différentes catégories. Ainsi, par exemple, on dit qu'il y a des régimes de groupes en vertu desquels les gains sont intégrés au salaire. Ici, le lecteur aimerait que l'on soit plus explicite car l'existence de tels régimes n'est pas évidente. Ainsi, il n'est pas surprenant de constater que, par la suite, on ne parle plus de cette catégorie de régimes.

Le chercheur intéressé par les questions d'évaluation du rendement et de rémunération au rendement restera un peu sur sa faim. Les auteurs ne procèdent pas à une étude en profondeur des recherches sur le sujet, et ce n'était probablement pas le but d'un tel rapport. D'autres livres, comme Merit Pay de R. Heneman, sont plus utiles à cet égard.

Cependant, dans l'ensemble, les quelques lacunes mentionnées n'enlèvent en rien les qualités du livre. Pay for Performance se lit très bien et fait, d'une façon simple, le point sur les questions d'actualité, d'évaluation du rendement et de rémunération au rendement. C'est un ouvrage que je n'hésite pas à recommander aux professionnels en gestion des ressources humaines qui s'intéressent à ces questions ainsi qu'aux gestionnaires préoccupés par la pertinence et l'efficacité de ces programmes.

Roland ThÉRIAULT 